\title{
LA TERMINOLOGÍA ENOLÓGICA DEL ESPAÑOL EN EL S. XIX ${ }^{1}$
}

\section{Francisca Bajo Santiago}

Universidad Rovira i Virgili

\section{RESUMEN}

La incorporación de la ciencia a la elaboración de vinos influye en el corpus léxico de la lengua española. Se crea una serie de términos que dan respuesta a las necesidades expresivas de la nueva ciencia vinícola. Este estudio analiza la terminología de la enología en el siglo XIX.

PALABRAS CLAVE: enología, terminología, siglo XIX.

\section{SUMMARY}

When science became a part of the process of wine production, the Spanish lexical corpus was correspondingly affected. Many terms were created which responded to the expressive needs of the new science of wine production. This study analyses the terminology of enology in the $19^{\text {th }}$ century.

KEY WORDS: enology, terminology, $19^{\text {th }}$ century.

\section{PRESENTACIÓN}

El presente estudio pone su atención en la «revolución» léxica que experimenta la terminología de la elaboración de vinos en el siglo XIX. La incorporación de la ciencia, especialmente de la química, a la vinificación lleva consigo la aparición de nuevos términos, lo que motiva una importante reestructuración del sistema terminológico de la enología.

1 Este trabajo forma parte del proyecto Catálogo de neologismos del léxico científico y técnico del s. XIX (MCYT, BFF 2001-2478). Agradezco la ayuda que el profesor C. Garriga me ha prestado en todo momento. 


\section{LA ENOLOGÍA ESPAÑOLA EN EL SIGLO XIX}

Según nos informa Muñoz Puelles², allá por el año 4000 a.C. los agricultores del próximo Oriente descubrieron que el zumo de la uva fermentado daba lugar a un líquido de interesante sabor y cualidades. Desde entonces hasta la actualidad los métodos de elaboración de vinos han experimentado notables cambios. El más significativo tuvo lugar a finales del siglo XVIII, cuando se incorporaron a la vinificación los nuevos conocimientos científicos, especialmente los de la química ${ }^{3}$. Por eso algunos investigadores sostienen que la enología es una rama de la ciencia química .

A finales del siglo XVIII se produce en Europa la «Revolución química». En ese momento reinaba en España un ambiente propicio a la «acogida favorable y fértil de las nuevas doctrinas» ${ }^{5}$, lo que favoreció la rápida traducción de los trabajos de Lavoisier, Morveau, Fourcroy, Berthollet... ${ }^{6}$ De la mano de la química moderna, la enología europea llega también en un momento muy propicio. Como anota Pan-Montojo, «la lectura de la literatura extranjera produjo un cambio substancial en el talante, preocupaciones y estilo de los tratadistas españoles» ${ }^{7}$. En este periodo destaca la figura de Carbonell y $\mathrm{Bravo}^{8}$, que mostró especial interés en aunar la industria con la ciencia.

2 Muñoz Puelles, V. (1998), El vino. Aroma, sabor, celebración, Valencia, Ediciones La Máscara, p. 11.

3 Peynaud, E. (1988), Le vin et les jours, París, Bordas, p. 88; RierA, S. (1993), «L'entrada de la ciència moderna a l'enologia» en GIRALT, E. (coord), Vinyes $i$ vins. Mil anys d'història, I, Barcelona, UB, 83-112, p. 83; PAN-MONTOJO, J. L. (1989), La vitivinicultura en España (1750-1988), Madrid, Ministerio de Agricultura, Pesca y Alimentación, p. 12.

4 Peynaud (1988), p. 117; Loubere, L. A. (1978), The red and white. A history of wine in France and Italy in the nineteenth century, Albany, State University of New York Press, p. 191.

5 Portela, E. (1998), La química en el siglo XIX, Madrid, Akal, p. 48-49.

6 GARRIGA, C. (1996), «Apuntes sobre la incorporación del léxico de la química al español: la influencia de Lavoisier», Documents pour l'histoire du française langue étrangere ou seconde, 18, 419-435; GARRIGA, C. (1997), «La recepción de la nueva nomenclatura química en español», Grenzgänge, 4, 8, 33-48.

7 PAN-Montojo (1989), p. 12.

8 RoF, M. C. (1971), Biografía y labor docente de Francisco Carbonell y Bravo, Barcelona, UB; NIETO, A. (1994), Ciència a Catalunya a l'inici del segle XIX: teoria i aplicacions tècniques a l'Escola de Química de Barcelona sota la direcció de Francesc Carbonell i Bravo (1805-1822), Barcelona, UB; NIETO, A. (1996), «Martí i Franquès, Carbonell i Bravo, i els usos de la nova química a la Catalunya il.lustrada» en VV. AA., Lavoisier $i$ els orígens de la química moderna, 200 anys després (1794-1994), Barcelona, SCHCT, 159-184; GUTIÉRREZ CuAdrado, J. (1998), «F. Carbonell y Bravo y su texto Curso analítico de química escrito en 
Aunque se había creado un clima favorable para el despegue de la ciencia enológica en España, se vio violentamente frenado por las circunstacias políticas del país. A mediados de siglo se observa un tímido despertar de la ciencia enológica española ${ }^{9}$, gracias a las aportaciones de estudiosos como Aragó, Bonet y Bonfill, Castellet o Hidalgo Tablada, que forman parte de esas generaciones intermedias que sentaron las bases de la recuperación científica en España ${ }^{10}$. El eslabón que unió estas dos etapas fue un discípulo de Carbonell, José Roura y Estrada ${ }^{11}$. Un lugar común en todas estas obras es la preocupación por el atraso en el que se encontraba la enología española y el deseo de mejorar los vinos, como revela De La Vega paradigmáticamente:

«Indicádose está con lo dicho, que no algo sino mucho, muchísimo falta que hacer y mucho que innovar, superior indudablemente á las facultades de la máxima parte de los cosecheros actuales, que por harto tiempo desgraciadamente, tendrán que contentarse con hacer lo que hacen, sin dejar por eso de ganar mucho con las instrucciones que encuentran $»^{12}$.

Este esfuerzo intelectual culmina, a finales del siglo XIX, en la gran etapa de esplendor de la enología española ${ }^{13}$, cuando «alcanzó especial resonancia la idea de que España estaba llamada a convertirse en la bodega del mun$d o{ }^{14}$, cuando se levantaron en la Rioja grandes bodegas al servicio de una

italiano por F. Mojon (1818)» en García TURZA, C. et al. (eds.), Actas del IV Congreso Internacional de Historia de la Lengua Española, II, Castilla y León, Junta de Castilla y León, 701-711.

9 PAn-Montojo (1989), p. 16-17.

10 LÓPEZ PiÑERO, J. M. (1967), «La literatura científica en la España contemporánea» en DíAz PlajA, G. (dir), Historia General de las Literaturas Hispánicas, VI, Barcelona, Vergara, 677-693, p. 680.

11 Martínez Nó, M. D. (1993), «El químic Josep Roura i Estrada (1791-1860) i la seva aportació a l'enologia», en GIRALT, E. (coord.), Vinyes $i$ vins. Mil anys d'història, I, Barcelona, UB, 193-206; PAn-Montojo (1989), p. 17; LusA, G. y RocA, A. (1997), «Ciència aplicada i industrialització a Catalunya. Les aportaciones de Josep Roura (1797-1860)», en ROURA, J. (1839), Memoria sobre los vinos y su destilacion y sobre los aceites, (ed. facsímil, Barcelona, ETSEIB, VII-XXXV).

12 De la VegA, D. (1861), Enología ó arte de hacer los vinos, Madrid, Imprenta de las Novedades, p. 5.

13 Pan-Montojo (1989), p. 19.

14 Pan-Montojo, J. L. (1994), La bodega del mundo. La vid y el vino en España (18001936), Madrid, Alianza, p. 11. 
rigurosa técnica de elaboración ${ }^{15}$ y cuando nació en Cataluña el primer cava español (1872) por iniciativa de Josep Raventós i Fatjó. Se multiplicó la publicación de textos enológicos ${ }^{16} \mathrm{y}$ se tradujeron con rapidez obras extranjeras, como el Tratado General de la vid y de los vinos (1892) de Viard:

«Al publicar esta edición española creemos, pues, haber realizado un trabajo, aunque modesto, de positiva utilidad y, por recompensa, aspiramos solo á que se reconozca nuestro buen deseo y se nos dispensen las faltas que hayan podido deslizarse, teniendo en cuenta que todo el trabajo de traducción y tirada le hemos llevado á término en tres meses muy escasos» ${ }^{17}$.

Finalmente, los premios recibidos por los vinos españoles en las Exposiciones Universales son un fiel testimonio de la llamada Edad de oro de la vitivinicultura hispánica ${ }^{18}$.

\section{FUENTES ENOLÓGICAS SELECCIONADAS}

Se afirma ${ }^{19}$ reiteradamente que la búsqueda de las fuentes y su selección es uno de los aspectos más importantes del trabajo terminológico, ya que condiciona el resultado de éste. Ahora bien ¿Cuántas obras deben seleccionarse y cuáles de ellas? Según Rondeau ${ }^{20}$ :

«Il n'existe aucun ensemble structuré de critères permettant d'evaluer la qualité linguistique de la documentation scientifique et technique, de sorte que traducteurs, terminologues, rédacteurs techniques et professeurs de LSP doivent établir individuellement et de façon empirique les éléments sur lesquels ils fondent leurs jugements».

15 Palacios, J. M. (1991), Historia del vino de Rioja, La Rioja, La prensa del Rioja, pp. 79-83.

16 Pan-Montojo (1989), p. 20.

17 Viard, E. (1892), Tratado general de la vid y de los vinos, Madrid, Direccion y Administracion.

18 GiRALt, O. (1993), «Els vins dels països catalans a les exposicions universals (Londres 1851-París 1900)» en GIRALT, E. (coord), Vinyes $i$ vins. Mil anys d'història, I, Barcelona, UB, 393-406.

19 DuBUc, P. (1992), Manuel pratique de terminologie, Québec, Linguatech, p. 38; CABRÉ, M. T. (1993), La terminología. Teoría, metodología, aplicaciones, Barcelona, Antártida/Empúries, p. 226; FEDOR DE DIEGO, A. (1995), Terminología.Teoría y práctica, Venezuela, Equinoccio, p. 102; RondeaU, G. (1984), Introduction à la terminologie, Québec, Gaëtan Morin, p. 52.

20 RondeAu (1984), p. 52. 
Para este estudio, teniendo en cuenta su importancia, su difusión y lo relevante de la fecha en cada caso, se han seleccionado los siguientes ocho manuales de enología, publicados entre 1803 y 1895 :

CAdeT-De-Vaux (1803), Arte de hacer el vino, (ed. facsímil, San Sebastián, Editorial Txertoa, 1983).

Boutelou, E. (1806), Idea de la práctica eonológica de Sanlucar de Barrameda, (ed. facsímil, Sanlúcar de Barrameda, Bodegas A. Babardillo, 1994).

CARBOnell, F. (1820), Arte de hacer y conservar el vino, (ed. facsímil, Catalunya, INCAVI, 1992).

BONET Y BONFILl, M. (1858), De la fermentacion alcohólica del zumo de la uva, Madrid, E. Aguado.

CASTEllet, B. (1865), Enologia española ó tratado sobre los vinos de España y su bonificacion, Barcelona, Imprenta de Gomez é Inglada.

LECANNU, R. L. (1871), Preceptos generales para la buena vinificacion, Madrid, Imprenta de la Sra. V. é Hijas de D. A. Peñuelas.

ARAGó, B. (1871), Tratado completo sobre el cultivo de la vid y elaboracion de vinos de todas clases, Madrid, Librería Central de D. Mariano Escribano.

MANso de ZúÑIGA, V. y DíAZ y Alonso, M. (1895), Conferencias enológicas, Madrid, Imprenta de los Hijos de M. G. Hernández.

\section{LA SELECCIÓN DE LOS TÉRMINOS ENOLÓGICOS}

Acotar exactamente en los textos especializados los términos que conforman la terminología de una disciplina puede resultar labor complicada, y ha suscitado muchas discusiones, como muestra claramente Fluck ${ }^{21}$. ¿Qué términos se deben seleccionar y cuáles no? A continuación se expone cómo se han solucionado aquí algunos casos que se han presentado a lo largo del proceso de selección. lizada.

3.1. Términos enológicos que se emplean fuera de la comunicación especia-

Como ejemplos uva, vino o fermentación. Se han seleccionado ya que pertenecen al ámbito de la enología, son utilizados por los enólogos y aparecen en fuentes especializadas. En el caso de fermentación se ha observado cómo

21 FluCK (1996), p. 47-59. 
el concepto asociado a este término va cambiando como consecuencia del conocimiento cada vez más riguroso que se tiene de los diferentes elementos que participan en este proceso, especialmente a partir de los estudios de Pasteur. Véanse los siguientes ejemplos:

«La fermentacion es el movimiento que se excita en el cubo en que está depositado el fruto, como tambien en las cubas en que se encierran el licor de las ubas» ${ }^{22}$.

«Se da el nombre de fermentaciones á las descomposiciones espontáneas que sufren las sustancias orgánicas cuando, privadas de la vida, se hallan bajo la influencia del aire, humedad y cierta temperatura» ${ }^{23}$.

\subsection{Términos enológicos que se emplean en otros ámbitos de especialidad.}

Se ha considerado que un «término pertenece a un ámbito si es usado en este ámbito» ${ }^{24}$. Esto no significa que no se tenga presente «el fenómeno de la aparición originaria de un término en una especialidad» ${ }^{25}$. Si se restringe el estudio de la terminología enológica a aquellas unidades que se emplean exclusivamente en el ámbito enológico se ofrece una imagen distorsionada, ya que precisamente no se presentarían muchos de los términos que sostienen esta nueva ciencia, como los de origen químico.

La terminología enológica del siglo XIX se caracteriza precisamente por la fusión de la terminología tradicional con la terminología moderna, ya sea originaria de la elaboración de vinos o proceda de otras ciencias. Así, en oficios, se encuentran términos como agricultor, bodeguero, pisador, trasegador o viñador, junto a otros como bacteriólogo, enologista, ingeniero agrónomo o químico. Algo similar ocurre con procesos y operaciones como asoleo, bazuqueo o destilación junto a eterificación, oxigenación, rectificación o volatilización.

22 CAdeT-De-VAuX (1803), p. 10.

23 ARAGÓ, B. (1871), p. 150.

24 CABRÉ, M. T. (1999), La terminología. Representación y comunicación, Barcelona, IULA, UPF, p. 138.

25 CABRÉ, M. T. (2001), «Consecuencias metodológicas de la propuesta teórica (I)», en CABré, M. T. y Feliu, J. (eds.), La terminología científico-técnica, Barcelona, IULA, UPF, 27-36, p. 33. 


\subsection{Términos sintagmáticos.}

Estos términos presentan grandes problemas a la hora de identificarlos y delimitarlos. Los estudiosos han presentado distintos criterios para ayudar a reconocer estas unidades ${ }^{26}$, como por ejemplo el establecimiento de una oposición. Es el caso de fermentación insensible/ fermentación sensible. Véanse los siguientes ejemplos documentados en la obra de Francisco Carbonell:

«Tapé la cántara, y la puse en un lugar fresco, á fin de que el vino durante la fermentacion insensible de todo el invierno se acabase de perfeccionar» ${ }^{27}$.

«su sabor aunque bastante fuerte y picante, era mucho mas agradable que inmediatamente despues de haber sufrido la fermentacion sensible; era algo mas dulce y pastoso, sin conservar nada del sabor del azucar» ${ }^{28}$.

Otro de los criterios argumentados es el empleo de determinados recursos tipográficos, como la cursiva o la mayúscula, para destacar ciertas unidades terminológicas. En los textos enológicos estudiados se ha hallado el uso de la letra cursiva para destacar términos como trabajar el lagar o gobernar el vino. Véanse los contextos en los que se han documentado:

«Á este fin se valen de unas varas ó estacas largas guarnecidas con puntas, cuyas estacas se van introduciendo y sacando sucesivamente de los lagares; ó bien hacen bajar los obreros en estos en donde estrujan y revuelven el orujo y el mosto; á lo cual llaman trabajar el lagar» ${ }^{29}$.

«En algunos paises (1) ponen en el vino encubado, mosto reciente de otras uvas frescas, á lo que llaman gobernar el vino» ${ }^{30}$.

Otro criterio importante es la sinonimia. En los textos especializados pueden encontrarse casos en los que se presentan como sinónimos un término simple y un término sintagmático. Así ocurre con albúmina y substancia parecida á la clara de huevo documentados en la obra de Manso y Díaz:

26 Auger, P. y Rousseau, L. (1987), Metodologia de la recerca terminològica, Barcelona, Departament de Cultura de la Generalitat de Catalunya, pp.26-27; CABRÉ (1993), pp. 304305; DuBuC, J. (1992), pp. 44-45; RonDEAU, G. (1984), pp. 79-80.

27 CARBONELl, F. (1820), p. 152.

28 CARbonell, F. (1820), p. 150.

29 CARBonell, F. (1820), p. 156.

30 ArAGÓ, B. (1871), p. 199. 
"Albúmina ó substancia parecida á la clara de huevo.- Sirve de alimento á los seres ó fermentos que producen la fermentacion» (Manso y Díaz 1895: 41).

\section{LA DATACIÓN DE LOS TÉRMINOS}

El estudio de la datación de los términos en las fuentes enológicas seleccionadas ha permitido establecer tres etapas diferentes:

1. Primera etapa (1803-1820). EL NACIMIENTO DE LA ENOLOGÍA ESPAÑOLA.

Se sitúan en esta etapa las obras de Cadet-de-Vaux (1803), Boutelou (1806) y Carbonell (1820). Se caracteriza por la convivencia de la terminología tradicional de la elaboración de vinos con la nueva terminología enológica. Así se han datado términos como bodega, colodra, prensa, uva, vendimiador o vino junto a otros como enología, enologista, eonológico/a, oenómetro u onólogo.

2. Segunda etapa (1858-1871). LA RECUPERACIÓN DE LA ENOLOGÍA ESPAÑOLA.

Se incluyen en esta etapa las obras de Bonet (1858), Castellet (1865) y Lecannu (1871). Desde la obra de Carbonell hasta mediados del siglo XIX se produce una ruptura en el desarrollo de los conocimientos enológicos en España como consecuencia de las circunstancias socio-políticas de la época. Esta situación empieza a cambiar a mediados de siglo con la publicación de nuevas obras, iniciándose así la recuperación de la ciencia enológica española. Esta etapa se caracteriza por la «importación» de términos químicos a la enología. Por ejemplo los que designan diferentes tipos de ácidos: ácido butírico, ácido caprílico, ácido capróico, ácido enántico, ácido láctico, ácido margárico, ácido succínico, ácido racémico o ácido tánico.

3. Tercera etapa (1871-1895). LA EDAD DE ORO DE LA ENOLOGÍA ESPAÑOLA.

Pertenecen a esta etapa las obras de Aragó (1871) y Manso y Díaz (1895). La terminología de este periodo se caracteriza por el importante número de términos que designan aparatos, instrumentos o máquinas como despalilladora pisadora, ebullioscopo de cuadrante de Brossard-Vidal, enobarómetro, lavadora de botellas, separadora del escobajo o vaporímetro de Plucker. 


\section{EL TRATAMIENTO DE LOS TÉRMINOS}

Se presentan a continuación unos ejemplos del estudio de datación realizado. El término ácido acético se ha documentado en Carbonell (1820), Bonet (1858), Castellet (1865), Aragó (1871) y Manso y Díaz (1895). Aragó emplea como sinónimo el término ácido del vinagre:

«Por ejemplo, se llama fermentacion alcohólica ó vinosa la que produce el alcohol ó el vino; fermentacion acética la que da orígen al ácido acético ó ácido del vinagre; láctica la que forma el ácido láctico» ${ }^{31}$.

El término ácido acético se introduce en el Diccionario de la Lengua Castellana de la Real Academia Española en la edición de 1884. Se acompaña de la marca técnica abreviada Quím. (Química) y se define de la siguiente manera bajo el sustantivo ácido:

«Principio inmediato no nitrogenado, que abunda mucho en la economía animal, y existe también en los vegetales. Se forma artificialmente por combinación del alcohol con el oxígeno. El vinagre es un ácido acético impuro y dilatado en agua».

Bajo el adjetivo acético, ca se ofrece una remisión, acompañada de la marca técnica Quím. (Química): «V. Ácido acético».

El término ácido acetoso se ha documentado en Bonet (1858) y Aragó (1871). Bonet presenta el término ácido aldehídico como sinónimo de ácido acetoso.

«Esto por lo que toca á los resultados finales, dejando á un lado por lo mismo el discutir si la accion se efectúa directamente, como lo indica esta ecuacion, ó si mas bien es debida á la formacion prévia del acétilo, pasando por los compuestos intermedios de óxido de acétilo, hidrato del mismo y ácido acetoso ó aldehídico hasta terminar en el acético, como admite el baron de Liebig» ${ }^{32}$.

«El oxígeno del aire ataca al alcohol del vino deshidrogenándole y convirtiéndose por esta causa en aldehido. Este absorve una nueva cantidad de oxígeno, y queda transformado en ácido acetoso, y este último, á medida que absorve más oxígeno, forma el ácido acético, base del vinagre» ${ }^{33}$.

El Diccionario de la Lengua Castellana de la Real Academia Española recoge el término ácido acetoso en la edición de 1884. Se acompaña de la mar-

31 ARAGÓ, B. (1871), pp. 150-151.

32 BONET, M. (1858), p. 29.

33 Aragó, B. (1871), p. 95. 
ca técnica abreviada Quím. (Química) y se define bajo el adjetivo ácido: «El menos oxigenado que el acético». Bajo el adjetivo acetoso, sa se presenta una remisión precedida de la marca Quím. (Química): «V. Ácido acetoso».

Se ha observado cómo Aragó (1871) y el diccionario académico siguen las propuestas que Lavoisier, Fourcroy, Berthollet y Morveau presentaron en el Metodo de la nueva nomenclatura química (1788), ya que el término formado con el sufijo -ico indica una saturación de oxígeno, mientras el término con el sufijo - oso señala una menor cantidad de oxígeno.

\section{CARACTERÍsticas de LA TERMinOlOGía ENOLÓGiCA EN EL S. XIX ${ }^{34}$}

Lo primero que llama la atención es la convivencia de la terminología tradicional con la terminología moderna. Así se han documentado en la obra de Manso y Díaz términos como zaranda y enomosgeno:

«También se emplean las zarandas, sobre todo cuando el raspón se separa, después de efectuar la pisa $\aleph^{35}$.

«El Dr. Carpené ha inventado un aparato llamado enosmogeno, para la separación del ácido acético. El enosmogeno está fundado en la diálisis, y dado lo poco que se ha generalizado y el no conocerle prácticamente, nos inducen á limitarnos á su enunciación $»^{36}$.

La incorporación de la ciencia a la elaboración de vinos trajo consigo la adopción de nuevos términos que designaban nuevos conceptos. La mayoría de ellos proceden de la química como éter enántico, pero también se encuentran términos originarios de la física como electricidad o de la biología como epispermo.

Destaca también la presencia de términos sintagmáticos que se han clasificado en dos grandes grupos términos sintagmáticos nominales y términos sintagmáticos verbales. El $99 \%$ de los términos sintagmáticos recogidos son nominales y la mayoría de ellos presentan la estructura de nombre + adjetivo (levadura elipsoidal) Dentro del grupo de los términos sintagmáticos verbales se encuentran las colocaciones terminológicas (espesarse el vino) y las locuciones verbales terminológicas (dar de baston).

34 Puede encontrarse más información en BAJO, F. (2003), La terminología enológica del español en el s. XIX, Tarragona, Universidad Rovira i Virgili (Tesis Doctoral).

35 Manso, V. y DíAZ, M. (1895), p. 38.

36 MAnso, V. y DíAz, M. (1895), p. 228. 
El estudio de los textos especializados ha demostrado que las unidades terminológicas sí están sujetas a la variación. En el caso de la terminología enológica del siglo XIX se ha constatado la presencia de variantes denominativas con respecto a la forma y variantes denominativas con respecto al uso. En el primer caso se distinguen variantes ortográficas (onólogo/enólogo), morfológicas (encubacion/encubamiento), léxicas (escobajo/raspa) y reducciones (máquina pisadora/pisadora).

En el segundo caso se encuentran términos acompañados de indicaciones diatópicas (crianza de los vinos), diatécnicas (azúcar de los frutos), diacrónicas (pastorización) y diafásicas (extractivo). Estas indicaciones suelen estar acompañadas de marcadores discursivos, como: «llaman», «llamamos», «recibe el nombre» $\mathrm{y}$ «con este nombre». Véanse a continuación, a modo de ejemplo, las indicaciones que acompañan a los términos situados entre paréntesis.

«En Málaga esta mezcla y la clarificacion subsiguiente tiene lugar en los almacenes de la ciudad, donde se efectua lo que allí llaman la crianza de los vinos» (Bonet 1858: 109)

«En el zumo de la uva y especialmente en todos los zumos azucarados extraidos de los frutos que contienen el azúcar especial, llamado por los químicos azúcar de los frutos ó incristalizable, la fermentacion alcohólica se desarrolla espontáneamente cuando al temperatura es de 15 á $20^{\circ}$ y ha estado en contacto el zumo con el aire atmosférico» (Aragó 1871: 152).

«Bajo este epígrafe comprenderemos dos operaciones, consideradas por algunos enólogos como semejantes, según la manera que tienen de exponerlas, y que realmente ofrecen diferencias apreciables, en cuanto al objeto que se persigue, y á los resultados obtenidos para establecer alguna diferencia entre una y otra. Nos referimos á la calefacción propiamente dicha ó pastorización, como ahora se llama, y á la del asoleado de los vinos» (Manso y Díaz 1895: 124).

«La parte líquida ó el zumo, que es la más importante, contiene: Agua. Azúcar de uva (glucosa). Goma. Mucílago. Pectina. Sustancias crasas. Aceites esenciales. Materias desconocidas, llamadas vulgarmente extractivo» (Aragó 1871: 228).

Los términos marcados diatópicamente son consecuencia del empleo de técnicas y procedimientos regionales que marcan y caracterizan un producto de una determinada zona con respecto al producto de otra. Se ha considerado que la presencia de estos términos geográficos personaliza la terminología del vino frente a otras terminologías en las que el empleo de «regionalismos» puede responder a otras causas. 


\section{CONCLUSIÓN}

La terminología enológica del español en el siglo XIX se caracteriza principalmente por la capacidad de combinar términos procedentes de la tradición con nuevos términos, ya sean originarios de la enología o de otras ciencias. En este sentido, constituye un ejemplo extraordinario de cómo pueden convivir elementos terminológicos de muy diverso origen sin que ello perjudique la comunicación en un campo de especialidad determinado. Al contrario, la convivencia de términos tradicionales y modernos, de origen enológico o de otros ámbitos científicos, posibilita la creación de una terminología enológica propia de especialistas, a la vez que garantiza la comunicación eficaz con los legos o con el grupo intermedio de trabajadores y artesanos desconocedores de la moderna ciencia, pero familiarizados con las denominaciones tradicionales. Este panorama debería completarse con el rastro etimológico de las novedades aparecidas en el siglo XIX y el estudio detallado de cada término, pero queda fuera del alcance de estas páginas.

\section{BIBLIOGRAFÍA}

ARAGÓ, B. (1871), Tratado completo sobre el cultivo de la vid y elaboracion de vinos de todas clases, Madrid, Librería Central de D. Mariano Escribano.

Auger, P. y Rousseau, L. (1987), Metodologia de la recerca terminològica, Barcelona, Departament de Cultura de la Generalitat de Catalunya.

BAJO, F. (2003), La terminología enológica del español en el s. XIX, Tarragona, Universidad Rovira i Virgili (tesis doctoral).

Bonet y BonfILl, M. (1858), De la fermentacion alcohólica del zumo de la uva, Madrid, E. Aguado.

Boutelou, E. (1806), Idea de la práctica eonológica de Sanlucar de Barrameda, (ed. facsímil, Sanlúcar de Barrameda, Bodegas A. Babardillo, 1994)

CABrÉ, M. T. (1993), La terminología. Teoría, metodología, aplicaciones, Barcelona, Antártida/Empúries.

CABRÉ, M. T. (1999), La terminología. Representación y comunicación, Barcelona, IULA, UPF.

CABRÉ, M. T. (2001), «Consecuencias metodológicas de la propuesta teórica (I)», en CABRÉ, M. T. y FELIU, J. (eds.), La terminología científico-técnica, Barcelona, IULA, UPF, 27-36, p. 33.

CAdeT-De-VAuX (1803), Arte de hacer el vino, (ed. facsímil, San Sebastián, Editorial Txertoa). 
CARBonell, F. (1820), Arte de hacer y conservar el vino, (ed. facsímil, Catalunya, INCAVI, 1992).

CASTEllet, B. (1865), Enologia española ó tratado sobre los vinos de España y su bonificacion, Barcelona, Imprenta de Gomez é Inglada.

De LA VeGA, D. (1861), Enología ó arte de hacer los vinos, Madrid, Imprenta de las novedades.

DuBuc, P. (1992), Manuel pratique de terminologie, Québec, Linguatech.

FEdor De Diego, A. (1995), Terminología.Teoría y práctica, Venezuela, Equinoccio.

FLUCK, HANS-R. (1996) Fachsprachen, Tübingen und Basel, A. Francke.

GARRIGA, C. (1996), «Apuntes sobre la incorporación del léxico de la química al español: la influencia de Lavoisier», Documents pour l'histoire du française langue étrangere ou seconde, 18, 419-435.

GARRIGA, C. (1997), «La recepción de la nueva nomenclatura química en español», Grenzgänge, 4, 8, 33-48.

GIRALT, O. (1993), «Els vins dels païssos catalans a les exposicions universals (Londres 1851-París 1900)», en GIRALT, E. (coord), Vinyes $i$ vins. Mil anys d'història, I, Barcelona, UB, 393-406.

Gutiérrez CuAdrado, J. (1998), «F. Carbonell y Bravo y su texto Curso analítico de química escrito en italiano por F. Mojon (1818)», en GARCíA TURZA, C. et al. (eds.), Actas del IV Congreso Internacional de Historia de la Lengua Española, II, Castilla y León, Junta de Castilla y León, 701-711.

LECANNU, R. L. (1871), Preceptos generales para la buena vinificacion, Madrid, Imprenta de la Sra. V. é Hijas de D. A. Peñuelas.

LÓPEZ PIÑERO, J. M. (1967), «La literatura científica en la España contemporánea», en DíAZ PlajA, G. (dir), Historia General de las Literaturas Hispánicas, VI, Barcelona, Vergara, pp. 677-693.

LOUBERE, L. A. (1978), The red and white. A history of wine in France and Italy in the nineteenth century, Albany, State University of Nueva York Press.

LusA, G. y RocA, A. (1997), «Ciència aplicada i industrialització a Catalunya. Les aportaciones de Josep Roura (1797-1860)», en ROURA, J. (1839), Memoria sobre los vinos y su destilacion y sobre los aceites, (ed. facsímil, Barcelona, ETSEIB, pp. VII-XXXV).

MAnso De ZúÑIGA, V. y DíAZ Y Alonso, M. (1895), Conferencias enológicas, Madrid, Imprenta de los Hijos de M. G. Hernández.

MARTÍNEZ NÓ, M. D. (1993), «El químic Josep Roura i Estrada (1791-1860) i la seva aportació a l'enologia» en GIRALT, E. (coord.), Vinyes $i$ vins. Mil anys d'història, I, Barcelona, UB, pp.193-206.

Morveau, Lavoisier, Berthollet y Fourcroy (1788), Metodo de la nueva nomenclatura química (Traducción de P. Gutiérrez Bueno), Madrid, Antonio Sancha. 
Muñoz Puelles, V. (1998), El vino. Aroma, sabor, celebración, Valencia, Ediciones La Máscara.

Nieto, A. (1994), Ciència a Catalunya a l'inici del segle XIX: teoria i aplicacions tècniques a l'Escola de Química de Barcelona sota la direcció de Francesc Carbonell $i$ Bravo (1805-1822), Barcelona, UB.

NiETO, A. (1996), «Martí i Franquès, Carbonell i Bravo, i els usos de la nova química a la Catalunya il.lustrada» en VV. AA. (eds.), Lavoisier $i$ els orígens de la química moderna, 200 anys després (1794-1994), Barcelona, SCHCT, pp.159-184.

Palacios, J. M. (1991), Historia del vino de Rioja, La Rioja, La prensa del Rioja.

PAN-Montojo, J. L. (1989), La vitivinicultura en España (1750-1988), Madrid, Ministerio de Agricultura, Pesca y Alimentación.

Pan-Montojo, J. L. (1994), La bodega del mundo. La vid y el vino en España (18001936), Madrid, Alianza.

Peynaud, E. (1988), Le vin et les jours, París, Bordas.

PORTELA, E. (1998), La química en el siglo XIX, Madrid, Akal.

ReAl ACAdEMia EsPañola (1884), Diccionario de la Lengua Castellana, Madrid, Imprenta de D. Gregorio Hernando, en REAL ACADEMIA EsPaÑola (2001), Nuevo Tesoro de la Lengua Española, Madrid, Espasa-Calpe.

RIERA, S. (1993), «L'entrada de la ciència moderna a l'enologia» en GIRALT, E. (coord.), Vinyes $i$ vins. Mil anys d'història, I, Barcelona, UB, pp. 83-112.

RoF, M. C. (1971), Biografia y labor docente de Francisco Carbonell y Bravo, Barcelona, UB.

RondeAU, G. (1984), Introduction à la terminologie, Québec, Gaëtan Morin.

VIARD, E. (1892), Tratado general de la vid y de los vinos, Madrid, Direccion y Administracion. 